THE SURVEY OF INCOME AND PROGRAM PARTICIPATION
THE REDESIGN OF THE SURVEY OF INCOME AND PROGRAM

PARTICIPATION

No. 203

Vicki J. Huggins

Donald P. Fischer

U. S. Bureau of the Census

U.S. Department of Commerce U.S. CENSUS BUREAU 


\title{
THE REDESIGN OF THE SURVEY OF INCOME AND PROGRAM PARTICIPATION ${ }^{1}$
}

\author{
Vicki J. Huggins and Donald P. Fischer \\ Vicki J. Huggins, Demographic Statistical Methods Division, Bureau of the Census, \\ Washington, DC 20233
}

\section{INTRODUCTION}

The Survey of Income and Program Participation (SIPP) is a complex survey conducted by the Census Bureau to provide information for federal policy makers and academic analysts on topics such as part-year poverty, government program participation and eligibility, health insurance coverage, and income distributions. The SIPP has been used as a multi-purpose survey providing cross-sectional, longitudinal and current event information. The primary goal of the survey though is a longitudinal one - select a nationally representative sample of households and follow the people in those households to assess changes in their characteristics over time. Quite often, the multi-purpose uses of the data have compromised the longitudinal uses in terms of sample size, data product availability and important longitudinal analyses.

In order to make the program more effective, the Census Bureau has recently undertaken a comprehensive reassessment and redesign of the SIPP program. This major redesign addresses problems and concerns that surfaced through SIPP's early years This reassessment is being done in conjunction with a redesign of the sample frame, stratification and selection that uses 1990 Decennial Census of Population and Housing information.

\section{CURRENT SIPP}

New national probability samples of households (panels) have been selected each year up through 1993. The original design was to have panels consisting of 20,000 households. Sample households are interviewed every 4 months for up to 32 months where data for the previous four months is collected. One round of interviewing covering the entire sample takes 4 months and is called a wave. Wave 1 is the first interview.

Either two or three panels may be in the field at any given time which provides an overlap design. The primary reason for using an overlap design is to enhance cross-sectional estimation. Longitudinal estimates can be obtained from one panel, however to reduce the effects of longitudinal nonsampling errors and increase sample size for cross-sectional estimates, overlap panels for the same time period can be combined. There have been exceptions to the typical design, primarily due to budget cut-backs.

This paper was written for presentation at the 1994 August American Statistical Association Meetings. It reports the general results of research undertaken by Census Bureau staff. The views expressed are attributable to the authors and do not necessarily reflect those of the Census Bureau. 
The questionnaire includes, in all waves of interviewing, a set of core questions on sources of income, amounts from these sources of income, labor force status and program participation. For most waves, sets of questions on specific topics - called topical modules - are added which augment the core data. Topical modules address issues such as assets and liabilities; school enrollment and financing; child care arrangements; taxes and many other issues.

For more information on the design of the current SIPP as well as the sources and magnitude of errors in estimates based on SIPP, refer to the SIPP Quality Profile (1990) by Jabine, King and Petroni.

\section{CONCERNS OF DATA USERS ON THE CURRENT SIPP}

The Census Bureau strived early on in the SIPP program to create an outreach process to constantly assess whether customer expectations were being met and whether the goals of the SIPP program were accurate goals. Many federal agencies, universities and research groups were involved in the development of the SIPP program goals, regular assessments, and design changes over the past 11 years.

These agencies, universities and research groups in conjunction with the Census Bureau identified many concerns and problems with the SIPP program. The Bureau tried to address some of these major concerns. For example, to improve the timeliness of data product release, major changes in content or procedures were introduced into SIPP only during designated windows of opportunity which were primarily at the start of a new panel. This allowed SIPP staff to catch up on processing and settle down into a reasonable schedule for data product preparation and release.

Many of the concerns raised to the Bureau on the SIPP program were not feasible to solve or address until now. Below is a list and description of the major concerns with the current SIPP program that were raised. Weinberg and Petroni [1992] present most of these as the critical issues that motivated many decisions for the 1990 redesigned SIPP. More detailed discussion is also found in the report from the Committee on National Statistics (CNSTAT) of the National Research Council (NRC) Committee Report on the future of the SIPP by Citro et al [1993].

\section{A. $\quad \underline{\text { Sample Size }}$}

The SIPP sample size was originally designed to be 20,000 households per panel. Under this scenario, cross-sectional estimates based on 2 or 3 panels could be 40,000 and 60,000 households. However, funding cutbacks resulted in sample sizes for many panels of only around 12,000 , providing combined panel sample sizes of up to 36,000 but longitudinal samples of only 12,000, less attrition and nonresponse. See table 1 for sample sizes of the 1984-1993 panels.

Small sample sizes in the SIPP have constrained meaningful analysis of many estimates for single panels, especially for subgroups such as Blacks and Hispanics or the poor. Longitudinal analyses have suffered even more since these estimates are greatly impacted by attrition which further reduces available sample. 


\section{B. Difficulty in Combining Panels}

Combining panels to obtain more reliable cross-sectional estimates can be difficult operationally. Also, data for the same time periods from overlapping panels were not always available concurrently.

\section{Need to Compensate Better for Nonresponse Bias}

At wave 1, the SIPP nonresponse rate is about $5-7 \%$. By wave 8 , the nonresponse rate is around $21 \%$. For example, see Table 2 for nonresponse rates from the 1991 panel. To the extent that nonrespondents are different from respondents, survey estimates will be biased. We have evidence of nonresponse bias for characteristics such as marital status, employment status and asset ownership [McArthur, et.al, 1986 and Sanchez, 1991]. We use edit, imputation and weighting procedures to try to compensate for some of this nonresponse bias but analysts are very concerned about the effectiveness of our efforts.

\section{Short Panel Lengths}

Panel lengths have normally been 32 months. But even this length of time has been too short to observe enough longitudinal phenomena for estimates such as spells of program participation, spells of poverty and spells of health insurance coverage.

\section{E. $\quad$ Need for an Improved Edit and Imputation System}

Users wanted SIPP to take greater advantage of the longitudinality of the SIPP for use in edit and imputation procedures. For example, information gathered at wave 1 could be used to edit/impute for wave 2. Also, better documentation of the edit and imputation methods was requested.

\section{F. Timeliness of Data Products}

Official Reports: Due to the complexity of SIPP data and other circumstances, official SIPP reports were not very timely or regular early in the program. After six standard quarterly reports from the 1984 panel, there has been no regular publication series for the core content on income and program participation. The small sample sizes made it difficult for analysts to make data comparisons for the quarterly reports since very few differences were supported statistically. Also, data processing stalled and unexplained anomalies in the data contributed to dropping the regular quarterly report series.

From 1986 - 1989, topical module reports were produced, then topical module and longitudinal data reports from 1989 - 1991. More recently, core cross-sectional and longitudinal reports on income, poverty status, and programs have been produced in addition to topical module reports. 
Data users though have recommended regular historical reports and a research report series be produced from the SIPP.

Microdata Files: $\quad$ There was a period of significant delays in release of microdata files. However, for some time now , this has not been a problem.

Recent improvements were made in the timeliness of microdata products, especially since 1990, with the goal of producing cross-sectional files from a panel approximately 6 months after data collection was completed in the field.

SIPP staff also initiated development of an electronic data dissemination program through SIPP-On-Call, an 'on-line' computer service for making data extractions from SIPP public use microdata files.

Certainly though, timeliness continues to be an area for targeted improvement in the SIPP redesign, for cross-sectional, longitudinal and topical module microdata.

\section{G. $\quad$ Content}

Few major concerns with the content of the SIPP were raised prior to redesign planning. When solicited, data users voiced concern over the fact that the topical module schedule appeared to be locked up, with little room for new topics.

To date, some research has been done to gain a better understanding of the concerns described in A-G above and investigate solutions to best address them. The next section provides a clear statement of the goals of the 1990 redesigned SIPP, an overview of the redesigned program and presents research and evaluation results utilized to make redesign decisions.

\section{4. $\quad$ REDESIGNED SIPP}

Starting with the 1996 panel, we will change the pattern of how we interview the 150,000 households typically interviewed each year. Instead of overlapping panels with 20,000 households each, we will combine all of the units into a single 50,000 household panel. Sample households will be interviewed every 4 months for about 4 years which will provide analysts with more longitudinal observations then the current design of $2 / 3$ years. A new panel will be introduced every 4 years, e.g., 2000 and 2004. The 1996 panel will also include an oversample of the low income population. The change in design beginning with the 1996 panel supports the primary goals of the SIPP: Producing longitudinal estimates of income and program participation, paying most attention to improving the information for people who are economically at risk, and improving the capability to respond to current policy needs in topical areas.

The redesign embraces all aspects of the SIPP program including sample design, questionnaire design and a move to computer assisted personal interviewing (CAPI), and longitudinal processing. A description of the redesign changes to address A-G in the previous section are described below in A-G, respectively. 


\section{A. $\quad$ Larger Sample Sizes Plus Oversampling}

SIPP data users constantly requested larger samples of the low income population. The Census Bureau conducted research on oversampling which showed potential reduction in variances for the number of Blacks in or near poverty, number of Hispanics in or near poverty, and number of persons in or near poverty to be $31 \%, 20 \%$ and $15 \%$ respectively. See Weller et.al. [1991]. Thus oversampling of the low income population is being implemented in the 1996 panel.

In addition to oversampling to increase sample sizes for the low income population, the issue of past small sample sizes in SIPP especially for longitudinal analysis is being addressed with the larger panels of 50,000 households. We will move from a cross-sectional sample base of about 36,000 to 50,000 and a longitudinal base of about 12,000 to 50,000 . This is quite a gain, in particular for longitudinal analyses.

\section{B. Non-overlapping Panels}

Designing overlap or non-overlap samples was the topic of many discussions. Two nonsampling errors that may effect panel samples and suggest an overlap design to reduce these errors are (1) time in sample bias and (2) bias due to nonresponse which increases over the life of a panel. Time in sample or panel bias occurs if the reporting by respondents is influenced by the survey process over time. Bias due to nonresponse occurs to the extent that nonrespondents are different from respondents and this has not been adequately adjusted for in sample estimates.

Having an overlap panel that can be combined for cross-sectional estimates has a dampening effect on these two nonsampling errors. Weinberg and Petroni (1992) discuss this in detail. Results from three separate studies on time in sample in the SIPP suggest little, if any, time in sample bias. Regarding attrition bias, Petroni and Weinberg observe that

" The overall attrition of longer panels will be only slightly higher than the final attrition rate for the current panels ( $25 \%$ versus $21 \%$ ).

Since most of the attrition occurs in the first year of a panel, overlapping panels after the second year would do little to reduce attrition. [This is in response to the CNSTAT's panel suggestion for a 2 year overlapping design.]

If panels are not combined (and they rarely are) the attrition rates for the overlapping design are identical to those for an abutting design."

These observations were key to the decision to redesign the SIPP with 4 year abutting panels with the obvious advantages of longer, larger panels for longitudinal analyses. However, this design still does not address the concern of the effects of attrition on both cross-sectional and longitudinal estimates. It simply supports the fact that overlapping panels do not offer a substantial dampening effect on attrition bias when compared to attrition bias of one panel. 


\section{Weighting and Imputation to Reduce Nonresponse Bias}

The Bureau has conducted a great deal of research on nonresponse in the SIPP attempting to 1) assess the differences in the responding and nonresponding universes, 2) estimate the effect of attrition on specific estimates such as monthly mean income amounts, poverty and program participation estimates and 3) investigate alternative imputation and weighting procedures to reduce nonresponse bias.

We have identified certain attributes that are associated with higher rates of nonresponse such as whether a person ever moved during the panel before attriting. [Jabine, et al., 1990] In fact, we are investigating whether a mover adjustment in weighting might reduce attrition bias. [Allen, et al., 1994] We also found that the current SIPP cross-sectional nonresponse weighting adjustment does reduce attrition bias for estimates of monthly mean and median income, but has no effect on program participation estimates. [Sanchez, 1991].

Recently completed research by Westat, Inc. and the Research Triangle Institute did not identify improved longitudinal nonresponse weighting methods for the SIPP. [Rizzo et al., 1994 and Folsom et al., 1994]

Imputation procedures that make use of the longitudinality of SIPP data have great promise for reducing nonresponse and attrition bias. Research was conducted by Pennel et al. [1993] to assess the effect of imputing missing interviews of data with information from interviews that were obtained before and after the missing interview - called carry-over imputation. Carry-over methods are much less complicated than other imputation methods and perform very well for many items.

At the Bureau, we applied the carry-over imputation method to the 1991 panel file. The number of eligible sample cases for weighting the panel increased by $8.5 \%$, a substantial increase. Plans are to continue using carry-over imputation in subsequent panels.

\section{4 Year Panels}

To meet the longitudinal needs of SIPP customers, it is clear that panel lengths have to be extended. The Census Bureau believes 4 year panels will address many of the limitations to data analysis inherent with 32 month panels.

\section{E. Improved Edit and Imputation System}

The Bureau now has comprehensive, user friendly documentation of the current SIPP edit and imputation system. [Pennel, 1993]. This documentation provides an explanation of the different types of nonresponse which occur in SIPP and the nature of the cross-sectional imputations and longitudinal edits performed to compensate for missing or inconsistent data. 
Computer Assisted Personal Interviewing (CAPI) will be used for the redesigned SIPP. This will permit consistency data checks in the interview that can reduce the level of postcollection edits and imputation. [CAPI will be discussed below in more detail.]

In the new processing system we will include the use of data collected in previous interviews to edit/impute data for current interviews. Longitudinal processing will allow us to reedit and impute data using all interviews to maintain longitudinal consistency.

\section{F. SIPP Data Products and Data Dissemination Programs}

The current proposal for SIPP redesign data products includes the following: Reports:

The Bureau will create a basic set of cross-sectional statistics for the SIPP cross-sectional reports which will include average monthly estimates of:

o Median household income

O Number of workers and their median earnings

○ Number or persons in poverty

O Persons with labor force activity

○ Participants in Government programs, such as AFDC, SSI, Medicare, Social Security, etc.

o Persons with health insurance coverage, by type of coverage

These statistics will be the basis for regular, official cross-sectional reports and/or fact sheets on the economic situation of Americans and their families starting with the first wave of 1996. The statistics should be released every four months after data collection and processing of the current wave as paper and/or electronic reports. produced.

In addition, topical module reports on disability, child care, wealth, etc. will continue to be

For the first calendar year report, we plan to produce longitudinal statistics such as

O Annual income and poverty estimates

O The effect of taxes/transfers on poverty

$\circ$ Poverty spells and transitions

o Median unemployment spells

O The number and characteristics of persons ever participating in government programs

o Median program participation spells

O The characteristics of persons with lapses in health insurance coverage

o Median spells without insurance

o Family and household transitions

The longitudinal reports will evolve as the length of observations grow. 
This comprehensive official report program should better serve data user needs.

Data files:

* Wave data files will contain the core data for that wave and the accompanying topical modules. These files will be edited and imputed based on previous wave information for the same individual.

* Longitudinal files will be prepared for calendar years 1, 1-2, 1-3, and 1-4. These files will be edited based on all the data available for that individual for the waves covered.

The longitudinal data products, in particular, will be more frequent which will better serve the longitudinal microdata needs of SIPP data users.

Data Dissemination:

Electronic data dissemination through SIPP-On-Call and/or Internet is being developed. SIPP-On-Call is a Census Bureau 'on-line' computer service for making data extractions from the SIPP public use microdata files. Cross-sectional files for the 1990 through 1993 panels are currently available. Plans are to include all topical module files for 1990 - 1993 as well. This form of data dissemination is a clear improvement in access to SIPP microdata and will continue to be developed for redesign data products.

Data users indicated they would like to have SIPP microdata available on CD-ROMs. The first CD-ROMs have already been produced for the 1987 and 1988 longitudinal panel files and we are working on the 1990 panel file. No cross-sectional data is planned for release on CD-ROMs, but we will continue creating CD-ROMS for the 1991-1993 and redesign longitudinal files.

\section{G. Content Changes and Enhancements}

No major changes in content were suggested by internal or external data users. The focus of redesign content development has been on developing questions and skip patterns to improve data quality. We have also been concerned with containing the scope of changes to not increase respondent burden above the current levels.

As presented, the Census Bureau has expended a great deal of effort to implement changes and enhancements into the SIPP program to satisfy requirements and expectations of customers. To ensure the feasibility of implementing the many design changes and the new processing for 1996, several field tests are underway to collect and process test data for redesign evaluation. The testing phase is described below.

\section{1990 REDESIGN TESTING}

In addition to sample and other methodological redesign, we are redesigning other aspects of the SIPP. Rather than simply automating the paper documents with CAPI, we are automating survey management and redesigning the data processing system. 
A CAPI instrument for the 1996 wave 1 interview was tested in April and May 1994. The test - called the Wave 1 pretest was primarily an operational test of training, interviewing procedures, data collection and data transmission.

The content test process began in January 1994, when Bureau staff began utilizing cognitive interviewing techniques to examine key questionnaire items. We modified the pretest version of the instrument described above including cognitively developed questions and implemented the modified version in a Wave 1 content field test. The areas of primary focus for the content test are revamping of the labor force questions, followup on amounts in the assets income questions, and clarification/ enhancement of general income questions and health insurance questions.

In November 1994, we will return to the Wave 1 content test interviewed households to conduct interviews for the Wave 2 content test.

Starting in February 1995, we will conduct a dress rehearsal of the complete redesign for Waves 1 and 2. This will include all operational aspects as well as weighting and data tabulations for evaluation purposes.

Through these planned testing activities, the Bureau expects an accurate and timely implementation of the 1996 panel.

\section{FUTURE EVALUATIONS}

With so many changes being implemented in the 1996 SIPP sample, the Bureau will need to continue its very ambitious research and evaluation program to assess the impact of the changes on data quality and customer expectations. Below are some areas that we believe should be considered for new - or renewed - research and evaluation by the Census Bureau.

- $\quad$ Evaluation of the dress rehearsal, CAPI Pretest and Content Test Results. Information such as response rates, problems with content and data quality issues should be summarized, evaluated and reported to data users;

Attrition. Attrition will increase more for a 4 year panel assuming current field procedures. Future research should focus on continuing to estimate attrition bias as well as improving weighting, imputation and other analytical methods to estimate or adjust for attrition bias. We also need to reassess data collection activities for handling nonrespondents and attritors to identify improvements;

- Undercoverage. We have substantial undercoverage of important subgroups such as young black males. Coverage for young black males age 20-24 was estimated to be around 65\% for May 1993 in the CPS. [Fenstermaker, 1993] SIPP rates are comparable. The Bureau should focus more resources to reduce undercoverage error; 
- $\quad$ Evaluation of cross-sectional and longitudinal estimates for data quality. It is critical that SIPP estimates be evaluated at the beginning of the 1996 panel for many reasons: benchmarking for the SIPP, data quality assessment as well as to help identify problem areas that must be corrected. We also need to look at the comparison of the redesigned data to administrative benchmarks and to the concurrent data collected from the 1993 panel.

- $\quad$ Longitudinal weighting and imputation research. The Bureau is working with Iowa State University to conduct additional research on longitudinal nonresponse adjustment. There is also an internal Bureau project to develop a longitudinal research plan. Continuing research in this area is critical since the goal of SIPP is now much more focused on longitudinal statistics.

- $\quad$ Assessment of Oversampling. As of the first interview in 1996, we will have information to begin assessing the success of oversampling. Evaluations should first focus on the success as of wave 1 in obtaining interviews for the oversampled low income population, then the success over time in maintaining these households in the survey. This will be important information in making decisions about oversampling in subsequent panels.

- $\quad$ Assessment of Processing System. Over the years, as resources permitted, we made changes to the system to satisfy new demands as much as possible. We are taking advantage of the CAPI system development to develop a new processing system that will be more efficient and better satisfy the needs of data users. With this major change, we will need to monitor our effectiveness in meeting these new processing goals.

The redesigned SIPP has the potential to provide more accurate and reliable national statistics than it has in the past with all the major changes in sample design, data collection, data processing and methods. The Census Bureau will continue to strive to assess and develop the SIPP program based on customer input and national issues.

\section{REFERENCES}

Allen, T. and Rita Petroni (1994), "Mover Noninterview Adjustment Research", Draft Paper for Presentation in the Survey Research Methods Section at the 1994 American Statistical Association Meeting.

Citro, C. and G. Kalton, Editors (1993), The Future of the Survey of Income and Program Participation, Panel to Evaluate the Survey of Income and Program Participation, Committee on National Statistics, National Research Council, National Academy Press.

Folsom, R. and M. Witt (1994), "Testing a New Attrition Nonresponse Adjustment Method for SIPP", Final Report for Census Bureau Contract No. 50-YABC-2-66024, Research Triangle Institute. 
Gbur, P., P. Cantwell and R. Petroni (1990), "Effect of Maximum Telephone Interviewing on SIPP Topical Module and Longitudinal Estimates", Proceedings of the Survey Research Methods Section, American Statistical Association.

Jabine, T., K. King and R. Petroni (1990), Survey of Income and Program Participation: Quality Profile, Bureau of the Census, Washington, D.C., U.S. Department of Commerce.

Lepkowski, J., S. Pennel, D. Miller and G. Kalton (1992), "Time in Panel Effects in the SIPP", Draft Final Report for Census Bureau JSA Contract 90-28, Ann Arbor, University of Michigan.

McArthur, E. and K. Short (1986), "Measurement of Attrition from the SIPP through the Fifth Wave of 1984 Panel", Internal Census Bureau Memorandum to Distribution List, April 10.

Pennel, S. (1993), "Cross-Sectional Imputation and Longitudinal Editing Procedures in the Survey of Income and Program Participation", Final Report for a Joint Statistical Agreement Between the Census Bureau and the University of Michigan No. 90-18.

Pennel, S., and J. Lepkowski (1992), "Panel Conditioning Effects in the Survey of Income and Program Participation", Draft paper presented at the 1992 American Statistical Association Meeting.

Rizzo, L., G. Kalton and M. Brick (1994), "Weighting Adjustments for Panel Nonresponse in the SIPP", Draft Final Report for Census Bureau Contract No. 50-YABC-266025, Westat Inc.

Sanchez, M. (1991), "SIPP 85: Evaluation of the Nonresponse Adjustment Procedure", Internal Census Bureau Memorandum for Singh, June 24.

Weinberg, D. and R. Petroni (1992), "The Survey of Income and Program Participation in the 1990's", SIPP Working Paper No. 9206, Bureau of the Census, Washington, D.C., U.S. Department of Commerce.

Weller, G., V. Huggins and R. Singh (1991), "Oversampling the Low-Income Population in the Survey of Income and Program Participation (SIPP)", Proceedings of the Survey Research Methods Section, American Statistical Association. 
Table 1. Household Sample Size by Panel

$\begin{array}{cc}\frac{\text { Panel }}{1984} & \text { Eligible Households } \\ 1985 & 20,897 \\ 1986 & 14,360 \\ 1987 & 12,425 \\ 1988 & 12,527 \\ 1989 & 12,725 \\ 1990 & 12,892 \\ 1991 & 23,627 \\ 1992 & 15,626 \\ 1993 & 21,577 \\ & 21,823\end{array}$

Table 2. 1991 Panel Household Nonresponse Rate by Wave

$\begin{array}{lc}\text { Wave } & \text { Nonresponse Rate (\%) } \\ 1 & 8.38 \\ 2 & 13.92 \\ 3 & 16.12 \\ 4 & 17.67 \\ 5 & 19.27 \\ 6 & 20.26 \\ 7 & 21.04 \\ 8 & 21,41\end{array}$

